\title{
Association of Polymorphisms of the Receptor for Advanced Glycation End Products Gene and Susceptibility to Sporadic Abdominal Aortic Aneurysm
}

\author{
Ye Yao, ${ }^{1}$ Junli Zhuang, ${ }^{1}$ You $\mathrm{Li}^{2}{ }^{2}$ Bao Jing, ${ }^{1} \mathrm{Hali} \mathrm{Li},{ }^{1} \mathrm{Jingbo} \mathrm{Li},{ }^{1}$ \\ Changgang Shao, ${ }^{1}$ Keshen $\mathrm{Li}^{2}{ }^{2}$ and Haiyang Wang ${ }^{1}$ \\ ${ }^{1}$ Division of Vascular Surgery, Department of General Surgery, The First Affiliated Hospital of Harbin Medical University, \\ Harbin, Heilongjiang 150001, China \\ ${ }^{2}$ Guangdong Key Laboratory of Age-Related Cardiac and Cerebral Diseases, Affiliated Hospital of Guangdong Medical College, \\ Zhanjiang 524001, China
}

Correspondence should be addressed to Haiyang Wang; haiyangwang811@163.com

Received 20 December 2014; Accepted 30 January 2015

Academic Editor: Christos V. Ioannou

Copyright (C) 2015 Ye Yao et al. This is an open access article distributed under the Creative Commons Attribution License, which permits unrestricted use, distribution, and reproduction in any medium, provided the original work is properly cited.

\begin{abstract}
Accumulating evidence has suggested that receptor for advanced glycation end products (RAGE) is involved in the development and progression of human abdominal aortic aneurysms (AAAs). However, the association between RAGE gene polymorphisms and AAA has not yet been determined. The present study was aimed at analyzing the potential association between the RAGE gene polymorphisms and AAAs. A cohort of 381 patients and 436 age-matched healthy controls were genotyped to detect the three RAGE polymorphisms ( $-374 \mathrm{~T} / \mathrm{A},-429 \mathrm{~T} / \mathrm{C}$, and G82S) using SNaPshot. Our study demonstrated a significant difference in the genotype and allele frequencies of the RAGE G82S polymorphism between the AAA patients and the controls. Further stratification by gender and smoking status revealed that the presence of the RAGE $82 S$ allele confers a higher risk for developing AAA in men and smokers. Moreover, AAA patients with the variant $82 \mathrm{~S}$ allele of RAGE presented with reduced serum soluble RAGE (sRAGE) production, and this decrease was more significant in men and smokers with AAA. Our study provides preliminary evidence that the $82 S$ allele of $R A G E$ is a risk factor for AAA. This new piece of knowledge regarding RAGE may be clinically important for the prevention and therapy of AAAs.
\end{abstract}

\section{Introduction}

Human abdominal aortic aneurysm (AAA), a major disease affecting the aorta, is the most common type of aneurysm in humans [1]. Rupture of the AAA is associated with high mortality, making aortic aneurysms a leading cause of death. The etiology of AAA involves chronic inflammation [2], loss of the extracellular protein elastin [3], increased neoangiogenesis [4], enhanced oxidative stress [5], and excessive degradation of the extracellular matrix (ECM) [6]; nevertheless, the precise mechanisms of AAA formation and progression are still elusive [7].

Receptor for advanced glycation end products (RAGE), a member of the immunoglobulin (Ig) superfamily, can interact with a broad range of ligands, including advanced glycation end products (AGEs), S100/calgranulins, high mobility group box-1 (HMGB1) protein, and $\beta$-amyloid peptide [8]. The binding of RAGE to its ligands not only stimulates the generation of reactive oxygen species (ROS) and the activation of nuclear factor kappa-B (NF- $\kappa \mathrm{B})$, mitogen-activated protein kinase (MAPK), and protein kinase $\mathrm{C}$ (PKC) but also induces cytokine production and inflammatory reactions, all of which are involved in the development and progression of AAAs [9]. In contrast, the soluble form of RAGE (sRAGE) can potentially bind to an AGE ligand thereby acting as a decoy, preventing the adverse effects of RAGE signaling [10]. The expression of RAGE and its ligand AGE was found to be significantly upregulated in human aneurysm tissues [11]. In vivo evidence also demonstrated that the inhibition of AGE signaling via targeted gene deletion of RAGE dramatically reduced the incidence of AAA in a mouse model [11]. 
Moreover, the aortic diameter was markedly abated in RAGEdeficient animals [11]. These lines of evidence have led us to hypothesize that RAGE may play a significant role in the pathogenesis of AAA.

The gene encoding $R A G E$ is located in the major histocompatibility complex locus on chromosome 6p21.3, and a number of polymorphisms have been identified [12]. The three most extensively studied polymorphisms include two SNPs in the promoter region (-429 T/C and $-374 \mathrm{~T} / \mathrm{A})$ and one SNP in exon 3 (G82S) of the RAGE gene. The $-429 \mathrm{~T} / \mathrm{C}$ and $-374 \mathrm{~T} / \mathrm{A}$ polymorphisms have been shown to exert significant effects on the transcriptional activity [13], and the G82S polymorphism, which occurs in the AGE binding domain, has been shown to have an enhanced ligandbinding affinity and leads to the increased ligand-stimulated activation of proinflammatory mediators [14]. The functional polymorphisms of the RAGE gene have been studied for their associations with various diseases, including Crohn's disease, systemic lupus erythematosus, Alzheimer's disease, multiple sclerosis, and cardiovascular diseases [15-20]. However, the association between the RAGE polymorphisms and AAA has not yet been determined. In light of the significant role of RAGE in the pathogenesis of AAA, the present study aimed to investigate the association of the RAGE polymorphisms $(-374 \mathrm{~T} / \mathrm{A},-429 \mathrm{~T} / \mathrm{C}$, and G82S) with AAA in a case-control study of the Han Chinese population.

\section{Materials and Methods}

2.1. Study Population. Our study consecutively recruited 381 patients (292 males and 99 females) with AAA from the Department of Vascular Surgery at the First Affiliated Hospital of Harbin Medical University from 2004 to 2013. The AAA diagnosis was confirmed using ultrasound scanning and spiral computed tomography, which can detect a dilatation of the abdominal aorta. The AAA was defined as a focal dilation of the abdominal aorta at least 50\% larger than the normal diameter. Patients with connective tissue disease, chronic renal failure, a known inflammatory or malignant disease, or familial AAAs were excluded from the study. A total of 436 age-matched control subjects (221 males and 215 females) from the same geographical area (Central Harbin) were recruited within the same period, and the aortic diameters of the control subjects were defined as a range from 19 to $22 \mathrm{~mm}$.

Written informed consent was obtained from each participant prior to enrollment in the study. This study was approved by the Ethics Committee of Harbin Medical University and was conducted according to the principles of the Declaration of Helsinki.

2.2. DNA Isolation and Genotyping. Genomic DNA was isolated from whole blood samples from all of the patients and controls by TIANamp Blood DNA Kit (Tiangen Biotech, Beijing, China) according to the manufacturer's instructions and stored at $-20^{\circ} \mathrm{C}$.

The RAGE gene polymorphisms - 374 T/A (rs1800624), $-429 \mathrm{~T} / \mathrm{C}$ (rs1800625), and G82S (rs2070600) were genotyped using the SNaPshot technique. The primers used were listed in Table 1. The genotyping was conducted using polymerase chain reaction (PCR) according to the manufacturer's protocol as it was described previously [21]. The experimental results were analyzed using GeneMapper 4.1 (Applied Biosystems, Foster City, CA, USA).

2.3. Enzyme-Linked Immunosorbent Assay (ELISA). Blood samples were collected as soon as the diagnosis was established. The sRAGE levels in the sera were determined in duplicate using Quantikine sandwich ELISA kits (R\&D Systems, Minneapolis, MN, USA) according to the manufacturer's instructions.

2.4. Assessment of the Aortic Diameter. The AAA size was determined as the greatest diameter of the infrarenal aorta using ultrasound. The maximum transverse and anteroposterior diameter of the infrarenal abdominal aorta was detected by an experienced vascular sonographer using a color Doppler ultrasound (GE Healthcare Technologies, Ultrasound, Milwaukee, WI, USA) with a $5 \mathrm{MHz}$ transducer. The reproducibility of aortic measurements is regularly assessed in the vascular surgery department. An abdominal aortic aneurysm is defined as a dilation of the aorta of at least 1.5 times its normal diameter or greater than a $3 \mathrm{~cm}$ diameter.

2.5. Statistical Analyses. The statistical analyses were conducted using SPSS, version 19.0 (IBM, Armonk, NY, USA). The clinical data are expressed as the means \pm standard deviation (SD) for the continuous variables and as the medians and percentage for the quantitative variables; a chi-squared test and Student's $t$-test were used to compare the variables between the two groups. The Hardy-Weinberg equilibrium (HWE) was tested using a chi-squared test to compare the observed genotype frequencies with the expected ones among the control subjects. Association between the polymorphism and the risk of AAA was evaluated using logistic regression analysis and was adjusted by age, gender, smoking, hypertension, diabetes mellitus, and dyslipidemia. The comparisons of the serum sRAGE levels among the different RAGE polymorphisms between the patients and controls were evaluated using Student's $t$-test for the normally distributed data or a Mann-Whitney $U$ test for the nonparametric data. The associations of the gene polymorphisms with the aortic diameter were analyzed using a multiple linear regression model. The criterion for significance was set at $P<0.05$ for all of the tests.

\section{Results}

3.1. Demographic Characteristics. The demographic characteristics of the participants are shown in Table 1 . The mean age of the patients (292 males and 89 females) was 69.8 years ( \pm 8.1 years), compared with 70.2 years $( \pm 7.5$ years) for the control subjects (221 males and 215 females). No significant differences were observed between the AAA patients and the controls with regard to age, diabetes, or hypertension. Significant gender and aortic diameter differences in the occurrence of AAA were observed. Compared with the controls, the AAA group included more smokers. Significant differences in the presence of dyslipidemia were also observed between the patients and controls. 
TABLE 1: Primers used in genotyping of RAGE -374 T/A, -429 T/C, and G82S polymorphisms.

\begin{tabular}{lcc}
\hline RAGE polymorphisms & Direction & Primers \\
\hline G82S (rs2070600) & Forward & $5^{\prime}$-GCTGGGGTTGAAGGCTTTTTCT-3' \\
\hline \multirow{2}{*}{$-374 \mathrm{~T} / \mathrm{A}(\mathrm{rs} 1800624)$ and $-429 \mathrm{~T} / \mathrm{C}(\mathrm{rs} 1800625)$} & Reverse & $5^{\prime}$-CCGGACAGAAGCTTGGAAGGTC-3' \\
\hline
\end{tabular}

3.2. Genotype and Allele Frequencies of the RAGE Polymorphisms in the AAA Patients and the Controls. The genotype and allele frequencies of the RAGE ( $-374 \mathrm{~T} / \mathrm{A},-429 \mathrm{~T} / \mathrm{C}$, and G82S) polymorphisms in the patients with AAA and the control individuals are shown in Table 2. A deviation from Hardy-Weinberg equilibrium for the RAGE polymorphisms was not found in the genotype distributions of the AAA patients and the control subjects (data not shown). The comparison of the genotype distributions between the AAA subjects and the control subjects using the $\chi^{2}$ test revealed that there was a statistical association $(P=0.020)$ between the G82S polymorphism of RAGE and the risk for AAA (Table 2). In a dominant model (82GG $+82 \mathrm{GS}$ versus $82 \mathrm{SS}$ ), no significant difference was detected between the AAA and control groups $(P=0.11)$. However, in a recessive model (82GG versus $82 \mathrm{GS}+82 \mathrm{SS})$, a significant difference was observed in the AAA group when compared with the controls $(\mathrm{OR}=0.67$, 95\% CI: $0.50-0.88, P=0.010)$. The prevalence of the $R A G E$ $82 \mathrm{~S}$ allele frequencies was significantly higher in the patients than in the controls ( $\mathrm{OR}=0.71,95 \% \mathrm{CI}: 0.57-0.88, P=$ $0.008)$. There were no statistically significant differences in the $-374 \mathrm{~T} / \mathrm{A}$ and $-429 \mathrm{~T} / \mathrm{C}$ genotypes and allele frequencies between the AAA cases and the control subjects (Table 2).

\subsection{Associations between the RAGE G82S Polymorphism and} the Demographic Characteristics. The associations between the RAGE G82S polymorphism and the demographic characteristics are shown in Table 3. In an analysis stratified by gender, an increased risk associated with the variant genotypes (82GS and 82SS) and alleles (82S) was found in the male patients $(P=0.025$ for the genotype and $P=0.012$ for the allele). When the sample was stratified according to smoking status, an increased risk associated with the variant genotypes and allele frequencies was more significant in the patients with AAA compared with the controls $(P=0.020$ for the genotype and $P=0.008$ for the allele). However, when the sample was stratified by either age, diabetes, or hypertension, no significant differences in the genotype or allele frequencies were detected between the AAA cases and the controls $(P>$ 0.05) (Table 4).

3.4. The Serum Levels of sRAGE according to the RAGE Genotypes. The serum levels of sRAGE were measured in 80 AAA patients and 80 healthy controls. As shown in Figure 1, the serum sRAGE levels were significantly lower in the AAA patients than in the controls $(918.58 \pm 152.36$ versus $625.39 \pm$ $121.55 \mathrm{pg} / \mathrm{mL}, P=0.035$ ) (Figure $1(\mathrm{a})$ ). Moreover, when the sample was stratified according to the RAGE genotypes, the serum sRAGE levels were significantly lower in the patients with the $82 \mathrm{GS}+82 \mathrm{SS}$ genotype than in those with the $82 \mathrm{GG}$ genotype $(852.25 \pm 133.66$ versus $586.32 \pm 108.23 \mathrm{pg} / \mathrm{mL}$, $P=0.025$ ) (Figure 1(b)). However, in the healthy controls carrying the $82 \mathrm{~S}$ allele, a slight but not significant decrease occurred. We also determined the serum RAGE levels in the AAA patients and controls who were stratified according to gender and smoking status. A significant decrease in the serum RAGE levels was found in the male patients $(756.18 \pm$ 117.15 versus $481.26 \pm 128.88 \mathrm{pg} / \mathrm{mL}, P=0.036)$ and the smoking patients $(665.86 \pm 109.33$ versus $453.82 \pm 115.72$ $\mathrm{pg} / \mathrm{mL}, P=0.012$ ) with the variant genotypes (82GG and 82GS) (Figures 1(c) and 1(d)).

3.5. Associations of the RAGE G82S Polymorphism with the $A A A$ Aortic Diameter. The associations of the RAGE G82S polymorphism with the AAA aortic diameter were explored, and the results are shown in Figure 2. The mean value of the AAA aortic diameter in the AAA patients with the variant genotypes (82GS and 82SS) was not significantly different from that of patients with the major 82GG genotype (55.8 \pm 10.8 versus $63.2 \pm 13.2 \mathrm{~mm}, P>0.05$ ) (Figure $2(\mathrm{a})$ ). Interestingly, when the AAA patients were stratified by gender and smoking status, a significant increase in the aortic diameter was found in the smoking patients with the variant genotypes $(82 \mathrm{GS}+82 \mathrm{SS})(57.8 \pm 9.8$ versus $70.2 \pm 12.5 \mathrm{~mm}, P=0.044)$ (Figure 2(b)). However, no difference in the aortic diameter was found in either the female or male patients with the variant genotypes $(54.1 \pm 8.2$ versus $60.8 \pm 9.3$ in female and $59.6 \pm$ 8.8 versus $64.5 \pm 11.5$ in male $\mathrm{mm}, P>0.05$ ) (Figure $2(\mathrm{c})$ ).

\section{Discussion}

In this hospital-based, case-control study, we demonstrated for the first time that the G82S polymorphism of RAGE is associated with AAA. Further stratification revealed that men or smokers with the RAGE 82S allele may run a higher risk of acquiring AAA. Additionally, the serum sRAGE levels were significantly lower in AAA patients with the variant $82 \mathrm{~S}$ allele than those carrying the major $82 \mathrm{GG}$ genotype.

As an inflammatory disease, the most significant pathologic feature of human AAA is the infiltration of inflammatory cells, including macrophages, monocytes, lymphocytes, and plasma cells, primarily to the outer part of the aorta [22]. Previous studies also demonstrated that oxidative stress is augmented during the process of AAA development [5]. Increased ROS is capable of enhancing inflammatory responses via the activation of $\mathrm{NF}-\kappa \mathrm{B}$, which is known to be a redox-sensitive transcription factor [23]. On the converse, activated inflammatory cells release ROS and further augment inflammatory responses. The engagement of RAGE with its ligands evokes the generation of intracellular 
TABLE 2: Characteristics of AAA cases and controls.

\begin{tabular}{lccc}
\hline Variables & AAA $(n=381)$ & Control $(n=436)$ & $P$ value \\
\hline Mean age (years) & $69.8 \pm 8.1$ & $70.2 \pm 7.5$ & 0.79 \\
Male/female & $292 / 89$ & $221 / 215$ & $<\mathbf{0 . 0 0 1}$ \\
Aortic diameter $(\mathrm{mm})$ & $56.3 \pm 7.5$ & $21.2 \pm 6.4$ & $<\mathbf{0 . 0 0 1}$ \\
Smokers, $n(\%)$ & $206(54.1)$ & $145(33.3)$ & $<\mathbf{0 . 0 0 1}$ \\
Diabetes, $n(\%)$ & $89(23.4)$ & $86(19.7)$ & 0.23 \\
Hypertension, $n(\%)$ & $147(38.6)$ & $155(35.6)$ & 0.86 \\
Dyslipidemia, $n(\%)$ & $185(48.6)$ & $152(34.9)$ & $<\mathbf{0 . 0 0 1}$ \\
\hline
\end{tabular}

Continuous data are presented as mean $\pm \mathrm{SD}$, median (range), or $n(\%)$

${ }^{\mathrm{a}} P$ values under 0.05 were indicated in bold font.

TABLE 3: Genotype and allele frequencies of RAGE polymorphisms between AAA patients and controls and corresponding ORs for AAA.

\begin{tabular}{|c|c|c|c|c|}
\hline Genotype and allele & $\begin{array}{l}\text { AAA patients } \\
(n=381)\end{array}$ & $\begin{array}{l}\text { Controls } \\
(n=436)\end{array}$ & OR $(95 \% \mathrm{CI})$ & $P$ value $^{\mathrm{a}}$ \\
\hline \multicolumn{5}{|l|}{-374T/A (rs1800624) } \\
\hline $\mathrm{TT}$ & $282(74.0)$ & $303(69.5)$ & & 0.31 \\
\hline $\mathrm{TA}$ & $88(23.1)$ & $115(26.4)$ & & \\
\hline $\mathrm{AA}$ & $11(2.9)$ & $18(4.1)$ & & \\
\hline $\mathrm{TT}+\mathrm{TA}$ versus AA & $370(97.1)$ & $418(95.9)$ & $1.45(0.68-3.11)$ & 0.34 \\
\hline TT versus TA + AA & $99(26.0)$ & $133(30.5)$ & $1.25(0.92-1.70)$ & 0.15 \\
\hline T allele & $652(85.6)$ & $721(82.7)$ & 1.00 & \\
\hline A allele & $110(14.4)$ & $151(17.3)$ & $1.24(0.95-1.62)$ & 0.11 \\
\hline \multicolumn{5}{|l|}{$-429 \mathrm{~T} / \mathrm{C}(\mathrm{rs} 1800625)$} \\
\hline $\mathrm{TT}$ & $297(78.0)$ & $344(78.9)$ & & 0.85 \\
\hline $\mathrm{TC}$ & $77(20.2)$ & $86(19.7)$ & & \\
\hline $\mathrm{CC}$ & $7(1.8)$ & $6(1.4)$ & & \\
\hline TT + TC versus CC & $374(98.2)$ & $430(98.6)$ & $0.75(0.25-2.24)$ & 0.60 \\
\hline TT versus TC + CC & $84(22.0)$ & $92(21.1)$ & $0.95(0.68-1.32)$ & 0.74 \\
\hline T allele & $671(88.1)$ & $774(88.4)$ & 1.00 & \\
\hline C allele & $91(11.9)$ & $98(11.6)$ & $0.93(0.69-1.27)$ & 0.66 \\
\hline \multicolumn{5}{|l|}{ G82S (rs2070600) } \\
\hline GG & $187(49.1)$ & $258(59.2)$ & & $0.020^{b}$ \\
\hline GS & $155(40.7)$ & $149(34.2)$ & & \\
\hline SS & $39(10.2)$ & $29(6.7)$ & & \\
\hline GG + GS versus SS & $342(89.8)$ & $407(93.3)$ & $0.63(0.38-1.03)$ & 0.11 \\
\hline GG versus GS + SS & $194(50.9)$ & $178(40.8)$ & $0.67(0.50-0.88)$ & 0.010 \\
\hline G allele & $529(69.4)$ & $665(76.3)$ & 1.00 & \\
\hline S allele & $233(30.6)$ & $207(23.7)$ & $0.71(0.57-0.88)$ & 0.008 \\
\hline
\end{tabular}

Data are presented as number (\%).

a Adjusted for age, gender, smoking, hypertension, diabetes mellitus, and dyslipidemia.

${ }^{\mathrm{b}} P$ values under 0.05 were indicated in bold font.

ROS and results in the activation of MAPK and NF- $\kappa \mathrm{B}$ signaling, which then triggers the release of inflammatory factors, cytokines, and chemokines, thereby contributing to the development and progression of AAA [9]. Previous studies demonstrated that RAGE is highly expressed in the infiltrating macrophages of human aneurysm tissues [11]. In vivo evidence also indicated that RAGE knockout mice are resistant to the formation of aneurysms [11], suggesting that RAGE signaling is involved in AAA development.
The observations that RAGE is present in aortic aneurysm tissue and that its expression is significantly increased in aneurysms have confirmed its involvement in the pathogenesis of AAA. Despite these advances, the RAGE alleles that contribute to the pathology of AAA remain undiscovered.

Several large genome-wide association (GWAS) studies have been performed [24-26]; however, the results are rather inconsistent, and the reported odds ratios (ORs) for the identified risk alleles in these studies are very low. These 


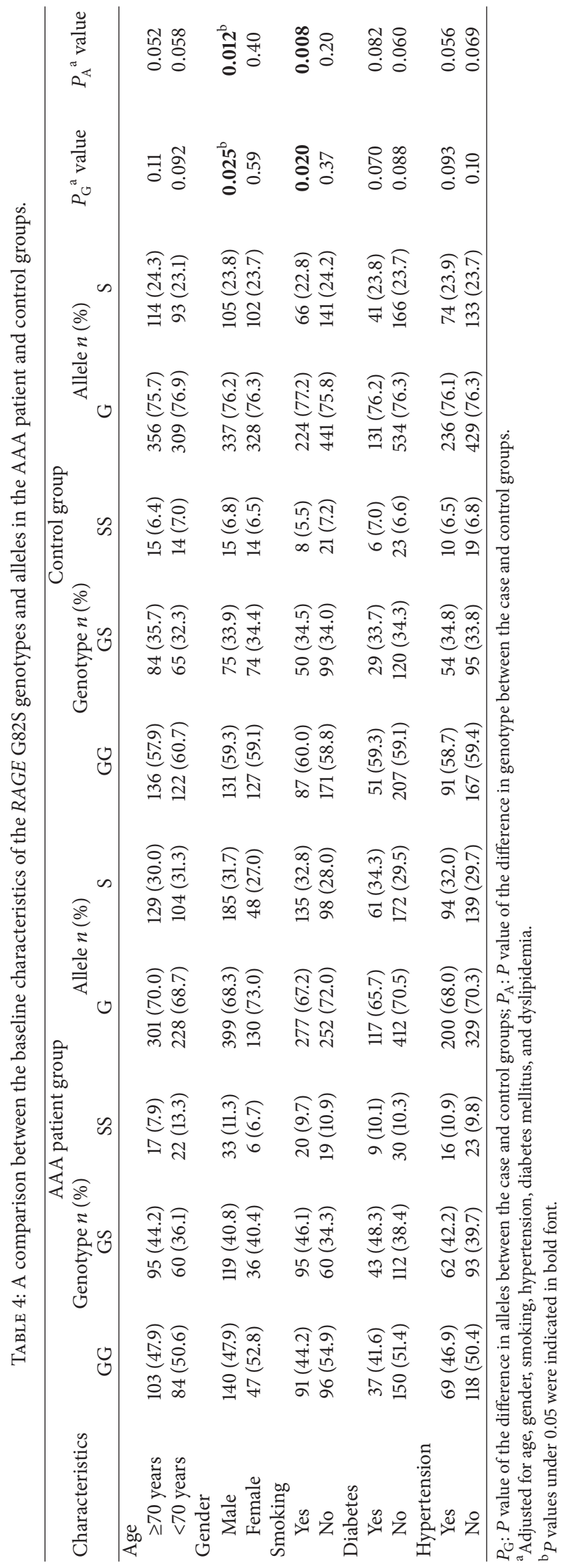




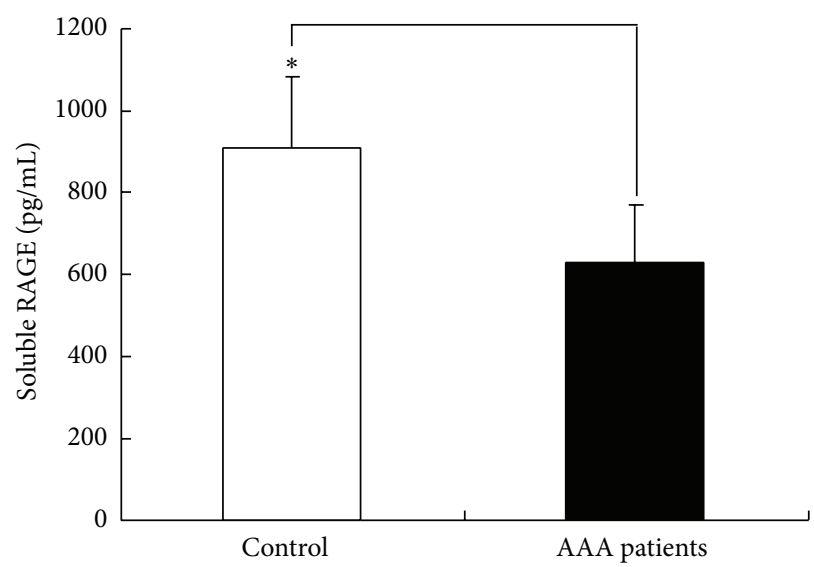

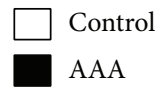

(a)

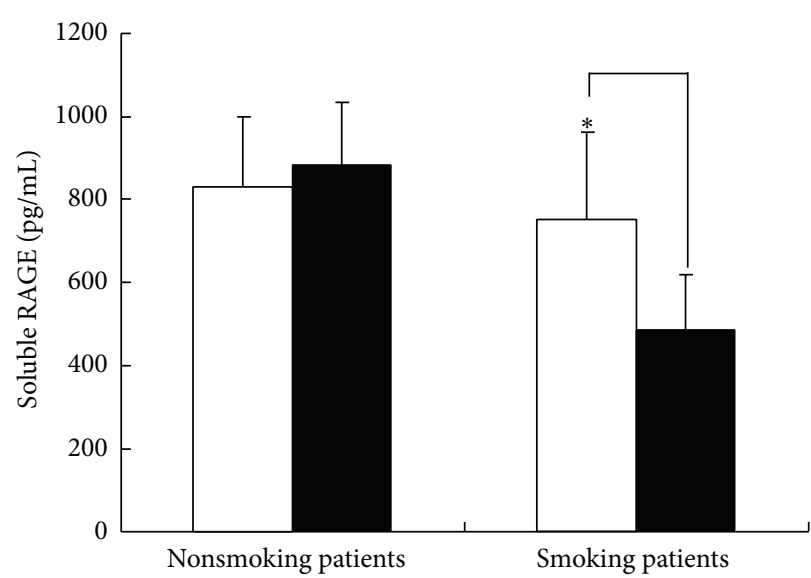

$\square$ GG
GS + SS

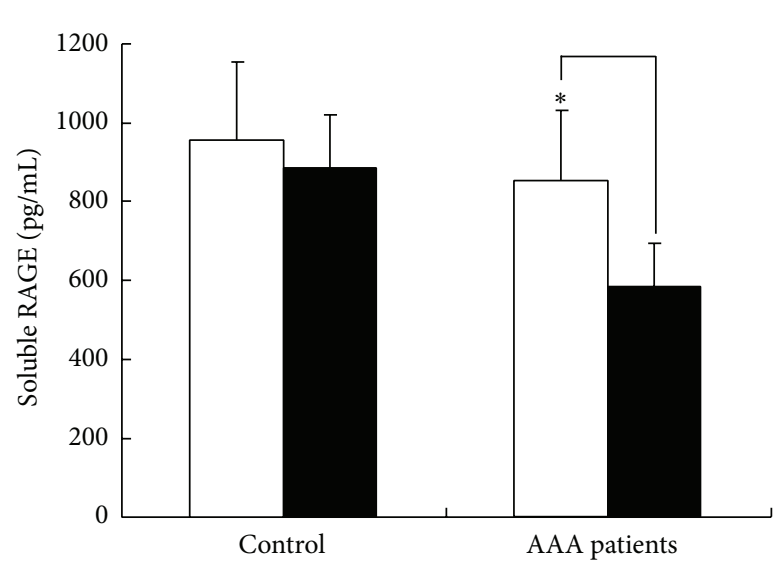

GG

$\mathrm{GS}+\mathrm{SS}$

(b)

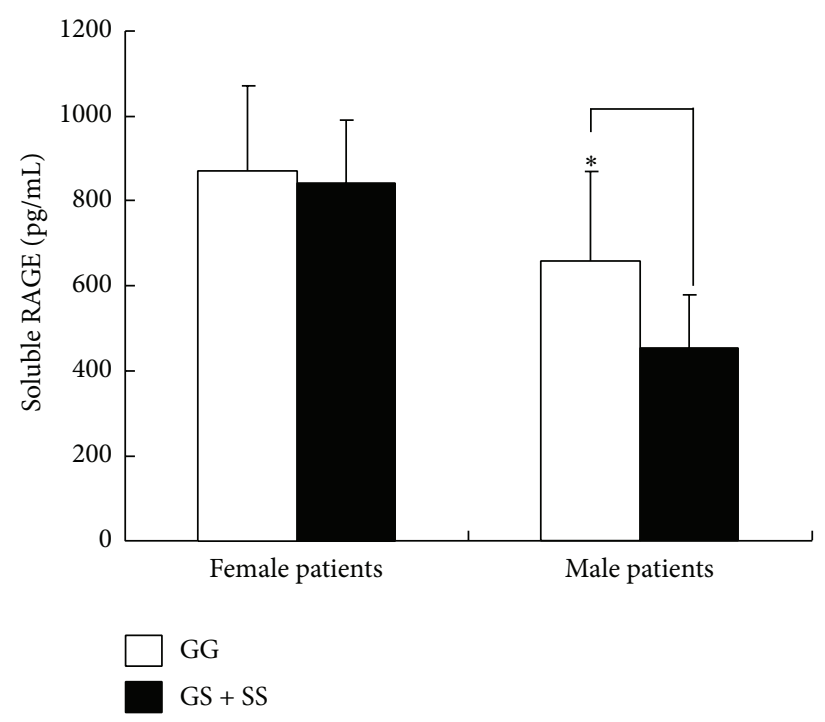

(d)

FIGURE 1: (a) The serum sRAGE levels in the AAA patients $(n=80)$ and the controls $(n=80)$. The blank box and the black box represent the serum sRAGE levels in the controls and the AAA patients, respectively. ${ }^{*} P=0.035$ when comparing the serum sRAGE levels between the AAA patients and the controls. (b) The serum sRAGE levels in the AAA patients and controls stratified according to the RAGE genotype. The blank box represents the 82GG genotype, and the black box represents the 82GS + 82SS genotype. ${ }^{*} P=0.025$. (c) The serum sRAGE levels in the AAA patients stratified according to smoking status and the RAGE genotype. ${ }^{*} P=0.012$. (d) The serum sRAGE levels in the AAA patients stratified according to gender and the RAGE genotype. ${ }^{*} P=0.036$. The serum sRAGE levels in the RRMS patients and the healthy individuals were measured using ELISA. The data are shown as the mean \pm SD. An asterisk indicates $P<0.05$.

discrepancies suggest that multiple risk loci are involved in the disease, and many are unidentified. In our casecontrol study, we show for the first time that the RAGE G82S polymorphism is associated with the risk of developing AAA. Our present findings imply that the G82S polymorphism of $R A G E$ may serve as a genetic marker for predicting AAA in high-risk populations.

The pathogenesis of AAA is the result of interactions between genetic predispositions and environmental factors. Epidemiological surveys demonstrated that the male sex is a risk factor for AAA, and males run a four- to fivetime greater risk than females [27]. Estrogen may play a protective role in AAA formation because estrogen was found to decrease inflammation and conversely correlate with MMP activity [28]. Being male is of course genetic, but it also affects environmental risk factors such as smoking, as well as hormonal levels, which are important mediators of aneurismal dilatation. In our present study, when the RAGE G82S genotype and allele frequency were further stratified by age, gender, smoking status, diabetes, and hypertension, an 




(a)



(b)

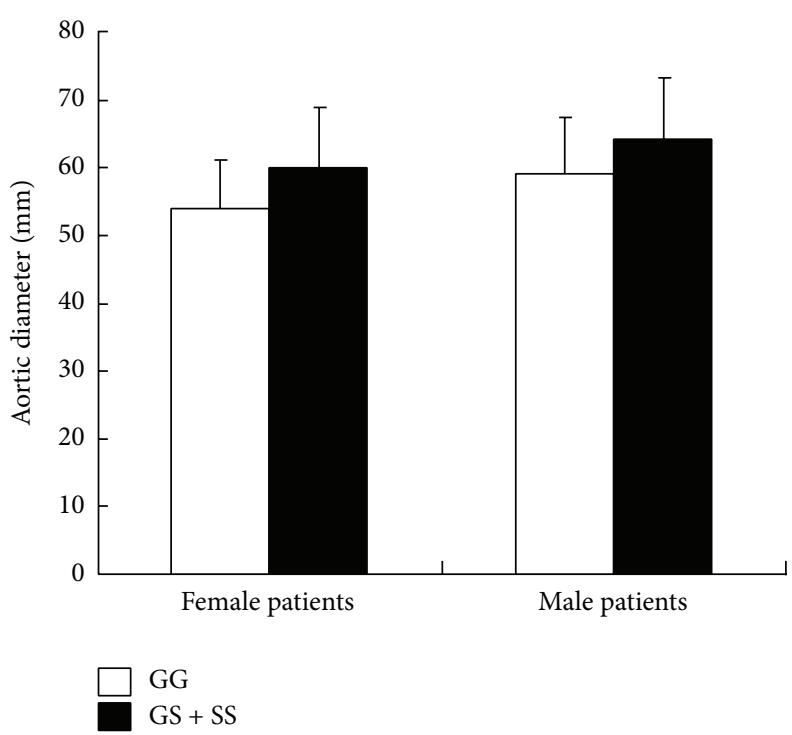

(c)

Figure 2: (a) The mean \pm SD values of the AAA diameter stratified according to the presence of the mutated allele. (b) The mean \pm SD values of the AAA diameter stratified according to smoking status and the presence of the mutated allele. ${ }^{*} P=0.044$. (c) The mean \pm SD values of the AAA diameter stratified according to gender and the presence of the mutated allele.

increased risk was found in the male and smoking subgroups of the AAA patients compared to the controls. Therefore, it is highly speculated that environmental risk factors, gender, and smoking may have interplay with the RAGE genetic predisposition in the present study.

The soluble form of RAGE (sRAGE) is a C-truncated secretory isoform of the receptor protein, which could work as a decoy receptor to abrogate RAGE signaling via inhibiting the binding of RAGE to its ligands. Therefore, the release of sRAGE may influence the regulation of RAGE-mediated functions in inflammatory events. The G82S polymorphism of $R A G E$ results in glycine being changed to serine at position 82 of the third exon encoding the RAGE protein and has been shown to be associated with both reduced serum levels of sRAGE and increased sRAGE signaling compared with the more common G allele $[19,29,30]$. This change in sRAGE signaling affects downstream gene expression through MAPK and NF- $\kappa \mathrm{B}$, both of which have been implicated in the inflammatory response in AAA [31]. In the present study, we showed that the serum levels of sRAGE were significantly decreased in AAA patients compared to the controls, supporting the potential role for the RAGE axis in the pathology of AAA. Moreover, we found that individuals carrying the mutated RAGE G82S S allele expressed lower sRAGE levels when compared with populations that carry the 82GG genotype. Given the key role of sRAGE in neutralizing circulating proinflammatory RAGE ligands, it is conceivable that the individuals with the $82 \mathrm{~S}$ allele expressing reduced serum sRAGE levels may result in an increase in the amount of ligands, which may cause the subsequent cellular responses. 
Therefore, the individuals with the RAGE $82 \mathrm{~S}$ allele will be more susceptible to RAGE ligand-induced inflammatory responses, which is a leading cause of AAA formation.

Accumulating epidemiological evidence confirmed that smoking is a significant contributing factor for AAA [32]. Current smokers have a significantly higher risk of developing AAA in both women and men than those who have never smoked [33]. Smoking also increases AAA growth, causing a greater risk of rupture and poor prognosis [34]. The formation and expansion of AAA involve widespread inflammation and extracellular matrix degradation. A recent study indicated that the AAA diameter was significantly associated with the hs-CRP plasma levels [35], which has evolved as an inflammatory risk marker of cardiovascular disease, suggesting that inflammation plays a potential pathogenic and causal role in the expansion of AAA. Other studies also suggested that an inhibitor against the platelet P2Y12 receptor reduces vascular inflammation and suppresses AAA expansion in $a p o E-/-$ mice [36]. These lines of evidence indicate that inhibiting inflammation may alleviate AAA expansion. Our study did not show any association of the polymorphisms with the aortic diameter; however, we confirmed a borderline association of the RAGE G82S polymorphism and the AAA diameter in smoking patients. Smoking as an environmental risk factor for AAA is very convincing and has been known for decades [32]. A study performed by UKSAT reported that active smoking is associated with an increased growth rate of $0.4 \mathrm{~mm}$ or $15 \%-20 \%$ greater than nonsmokers [34]. Additionally, cigarette smoke is an important exogenous source of reactive glycation products that can promote the formation of AGEs, thereby leading to RAGE-AGE axis-mediated signaling [37]. Based on the findings in our present study, it is conceivable that environmental exposure factors, in particular smoking, and genetic factors (82S allele of RAGE) may work synergistically in the pathophysiology of AAA expansion.

Despite the evidence, our study has several limitations that should be accounted for when interpreting the results. First, the study sample was not sufficiently large, which may lead to nonrepresentative results. Therefore, the preliminary findings of the present study are not definitive and must be replicated in a larger population. Second, selection bias in the patient or control populations cannot be entirely excluded. Additionally, the information regarding the important risk factors of AAA, such as smoking, was gathered from a selfreporting questionnaire, which may also introduce information bias. Third, other risk factors in the study group, such as Chlamydia pneumoniae infection and immune responses, which have been suggested to play a pivotal role in the pathogenesis of AAA, may have complicated the association between the RAGE polymorphisms and AAA. Finally, other functional polymorphisms located in the major histocompatibility complex locus may also affect the expression of sRAGE and contribute to the AAA risk, and their combined effects should not be neglected for predicting the occurrence, severity, and outcome of AAA. Larger patient and control cohorts from different ethnic backgrounds will be needed to confirm the association of the RAGE gene polymorphism with AAA in other populations.
In conclusion, our study is the first to show a significant association between the RAGE G82S polymorphism and the risk of developing AAA. Our findings support the notion that the G82S polymorphism of RAGE contributes to the development of AAA. In particular, males or smokers carrying the $82 S$ allele of $R A G E$, which is associated with decreased sRAGE levels, may run a higher risk of developing AAA. Our study may provide clues for use in the evaluation of individual susceptibility to AAA and to explore effective measures for the control and prevention of AAA. However, this is a preliminary study, and the results need to be confirmed in a larger cohort.

\section{Conflict of Interests}

The authors declare that there is no conflict of interests regarding the publication of this paper.

\section{Authors' Contribution}

Ye Yao and Junli Zhuang contributed equally to this paper.

\section{Acknowledgments}

This work was supported by funding from the National Nature Science Foundation of China (Grant nos. 81350026, 31171219, 81271213, 81070878, 81271214, 81300929, and 81471294); the Natural Science Foundation of Heilongjiang Province (H201344); the Medical Scientific Research Foundation of Guangdong Province, China (A2014483 and B2014305); and the PhD Start-Up Fund of Guangdong Medical College (B2012024).

\section{References}

[1] J. M. Reilly and M. D. Tilson, "Incidence and etiology of abdominal aortic aneurysms," Surgical Clinics of North America, vol. 69, no. 4, pp. 705-711, 1989.

[2] E. Choke, G. Cockerill, W. R. W. Wilson et al., "A review of biological factors implicated in abdominal aortic aneurysm rupture," European Journal of Vascular \& Endovascular Surgery, vol. 30, no. 3, pp. 227-244, 2005.

[3] M. D. Huffman, J. A. Curci, G. Moore, D. B. Kerns, B. C. Starcher, and R. W. Thompson, "Functional importance of connective tissue repair during the development of experimental abdominal aortic aneurysms," Surgery, vol. 128, no. 3, pp. 429$438,2000$.

[4] M. M. Thompson, L. Jones, A. Nasim, R. D. Sayers, and P. R. F. Bell, "Angiogenesis in abdominal aortic aneurysms," European Journal of Vascular and Endovascular Surgery, vol. 11, no. 4, pp. 464-469, 1996.

[5] J. Zhang, J. Schmidt, E. Ryschich, M. Mueller-Schilling, H. Schumacher, and J. R. Allenberg, "Inducible nitric oxide synthase is present in human abdominal aortic aneurysm and promotes oxidative vascular injury," Journal of Vascular Surgery, vol. 38, no. 2, pp. 360-367, 2003.

[6] N. P. Kadoglou and C. D. Liapis, "Matrix metalloproteinases: contribution to pathogenesis, diagnosis, surveillance and treatment of abdominal aortic aneurysms," Current Medical Research and Opinion, vol. 20, no. 4, pp. 419-432, 2004. 
[7] I. Hinterseher, R. Erdman, J. R. Elmore et al., "Novel pathways in the pathobiology of human abdominal aortic aneurysms," Pathobiology, vol. 80, no. 1, pp. 1-10, 2013.

[8] T. Chavakis, A. Bierhaus, and P. P. Nawroth, "RAGE (receptor for advanced glycation end products): a central player in the inflammatory response," Microbes and Infection, vol. 6, no. 13, pp. 1219-1225, 2004

[9] K. Fukami, S. Yamagishi, and S. Okuda, "Role of AGEsRAGE system in cardiovascular disease," Current Pharmaceutical Design, vol. 20, no. 14, pp. 2395-2402, 2014.

[10] I. H. Park, S. I. Yeon, J. H. Youn et al., "Expression of a novel secreted splice variant of the receptor for advanced glycation end products (RAGE) in human brain astrocytes and peripheral blood mononuclear cells," Molecular Immunology, vol. 40, no. 16, pp. 1203-1211, 2004.

[11] F. Zhang, K. C. Kent, D. Yamanouchi et al., "Anti-receptor for advanced glycation end products therapies as novel treatment for abdominal aortic aneurysm," Annals of Surgery, vol. 250, no. 3, pp. 416-423, 2009.

[12] R. Y. L. Zee, J. R. Romero, J. L. Gould, D. A. Ricupero, and P. M. Ridker, "Polymorphisms in the advanced glycosylation end product-specific receptor gene and risk of incident myocardial infarction or ischemic stroke," Stroke, vol. 37, no. 7, pp. 1686$1690,2006$.

[13] B. I. Hudson, M. H. Stickland, T. S. Futers, and P. J. Grant, "Effects of novel polymorphisms in the RAGE gene on transcriptional regulation and their association with diabetic retinopathy," Diabetes, vol. 50, no. 6, pp. 1505-1511, 2001.

[14] M. A. Hofmann, S. Drury, B. I. Hudson et al., "RAGE and arthritis: the G82S polymorphism amplifies the inflammatory response," Genes and Immunity, vol. 3, no. 3, pp. 123-135, 2002.

[15] H. A. Martens, H. L. A. Nienhuis, S. Gross et al., "Receptor for advanced glycation end products (RAGE) polymorphisms are associated with systemic lupus erythematosus and disease severity in lupus nephritis," Lupus, vol. 21, no. 9, pp. 959-968, 2012.

[16] J. Däbritz, F. Friedrichs, T. Weinhage et al., "The functional 374T/A polymorphism of the receptor for advanced glycation end products may modulate Crohn's disease," The American Journal of Physiology-Gastrointestinal and Liver Physiology, vol. 300, no. 5, pp. G823-G832, 2011.

[17] W. Lu, B. Feng, G. Xie, and F. Liu, "Association of AGER gene G82S polymorphism with the severity of coronary artery disease in Chinese Han population," Clinical Endocrinology (Oxford), vol. 75, no. 4, pp. 470-474, 2011.

[18] X. Cui, H. Chen, X. Hou, S. Wang, S. Jayaram, and Z. Zheng, "Polymorphism of the RAGE affects the serum inflammatory levels and risk of ischemic stroke in a chinese population," Cellular Physiology and Biochemistry, vol.32, no. 4, pp. 986-996, 2013.

[19] K. Li, B. Zhao, D. Dai et al., "A functional p.82G $>$ S polymorphism in the RAGE gene is associated with multiple sclerosis in the Chinese population," Multiple Sclerosis, vol. 17, no. 8, pp. 914-921, 2011.

[20] K. Li, D. Dai, B. Zhao et al., "Association between the RAGE G82S polymorphism and Alzheimer's disease," Journal of Neural Transmission, vol. 117, no. 1, pp. 97-104, 2010.
[21] Y. Li, F. Liao, X. J. Yin et al., "An association study on ADAM10 promoter polymorphisms and atherosclerotic cerebral infarction in a chinese population," CNS Neuroscience and Therapeutics, vol. 19, no. 10, pp. 785-794, 2013.

[22] J. S. Lindholt and G.-P. Shi, "Chronic inflammation, immune response, and infection in abdominal aortic aneurysms," European Journal of Vascular and Endovascular Surgery, vol. 31, no. 5, pp. 453-463, 2006.

[23] T. Finkel and N. J. Holbrook, "Oxidants, oxidative stress and the biology of ageing," Nature, vol. 408, no. 6809, pp. 239-247, 2000.

[24] J. R. Elmore, M. A. Obmann, H. Kuivaniemi et al., "Identification of a genetic variant associated with abdominal aortic aneurysms on chromosome 3 p12.3 by genome wide association," Journal of Vascular Surgery, vol. 49, no. 6, pp. 1525-1531, 2009.

[25] S. Gretarsdottir, A. F. Baas, G. Thorleifsson et al., "Genomewide association study identifies a sequence variant within the DAB2IP gene conferring susceptibility to abdominal aortic aneurysm," Nature Genetics, vol. 42, no. 8, pp. 692-697, 2010.

[26] M. J. Bown, G. T. Jones, S. C. Harrison et al., "Abdominal aortic aneurysm is associated with a variant in low-density lipoprotein receptor-related protein 1," The American Journal of Human Genetics, vol. 89, pp. 619-627, 2011.

[27] D. J. Katz, J. C. Stanley, and G. B. Zelenock, "Gender differences in abdominal aortic aneurysm prevalence, treatment, and outcome," Journal of Vascular Surgery, vol. 25, no. 3, pp. 561$568,1997$.

[28] A. Laser, A. Ghosh, K. Roelofs et al., "Increased estrogen receptor alpha in experimental aortic aneurysms in females compared with males," Journal of Surgical Research, vol. 186, no. 1, pp. 467-474, 2014.

[29] D. J. Smith, S. T. Yerkovich, M. A. Towers, M. L. Carroll, R. Thomas, and J. W. Upham, "Reduced soluble receptor for advanced glycation end-products in COPD," European Respiratory Journal, vol. 37, no. 3, pp. 516-522, 2011.

[30] Y. Jang, J. Y. Kim, S.-M. Kang et al., "Association of the Gly82Ser polymorphism in the receptor for advanced glycation end products (RAGE) gene with circulating levels of soluble RAGE and inflammatory markers in nondiabetic and nonobese Koreans," Metabolism, vol. 56, no. 2, pp. 199-205, 2007.

[31] R. P. Young, B. A. Hay, and R. J. Hopkins, "Does RAGE protect smokers from COPD?” European Respiratory Journal, vol. 38, no. 3, pp. 743-744, 2011.

[32] M. Bjorck and A. Wanhainen, "Pathophysiology of AAA: heredity vs environment," Progress in Cardiovascular Diseases, vol. 56, no. 1, pp. 2-6, 2013.

[33] O. Stackelberg, M. Björck, S. C. Larsson, N. Orsini, and A. Wolk, "Sex differences in the association between smoking and abdominal aortic aneurysm," The British Journal of Surgery, vol. 101, no. 10, pp. 1230-1237, 2014.

[34] A. R. Brady, S. G. Thompson, F. G. R. Fowkes, R. M. Greenhalgh, J. T. Powell, and Participants UKSAT, "Abdominal aortic aneurysm expansion: risk factors and time intervals for surveillance," Circulation, vol. 110, no. 1, pp. 16-21, 2004.

[35] J. de Haro, F. Acin, S. Bleda, C. Varela, F. J. Medina, and L. Esparza, "Prediction of asymptomatic abdominal aortic aneurysm expansion by means of rate of variation of C-reactive protein plasma levels," Journal of Vascular Surgery, vol. 56, no. 1, pp. 4552, 2012. 
[36] O. Liu, L. Jia, X. Liu et al., “Clopidogrel, a platelet P2Y12 receptor inhibitor, reduces vascular inflammation and angiotensin II induced-abdominal aortic aneurysm progression," PLoS ONE, vol. 7, no. 12, Article ID e51707, 2012.

[37] C. Cerami, H. Founds, I. Nicholl et al., "Tobacco smoke is a source of toxic reactive glycation products," Proceedings of the National Academy of Sciences of the United States of America, vol. 94, no. 25, pp. 13915-13920, 1997. 



Submit your manuscripts at

http://www.hindawi.com

Journal of
Signal Transduction
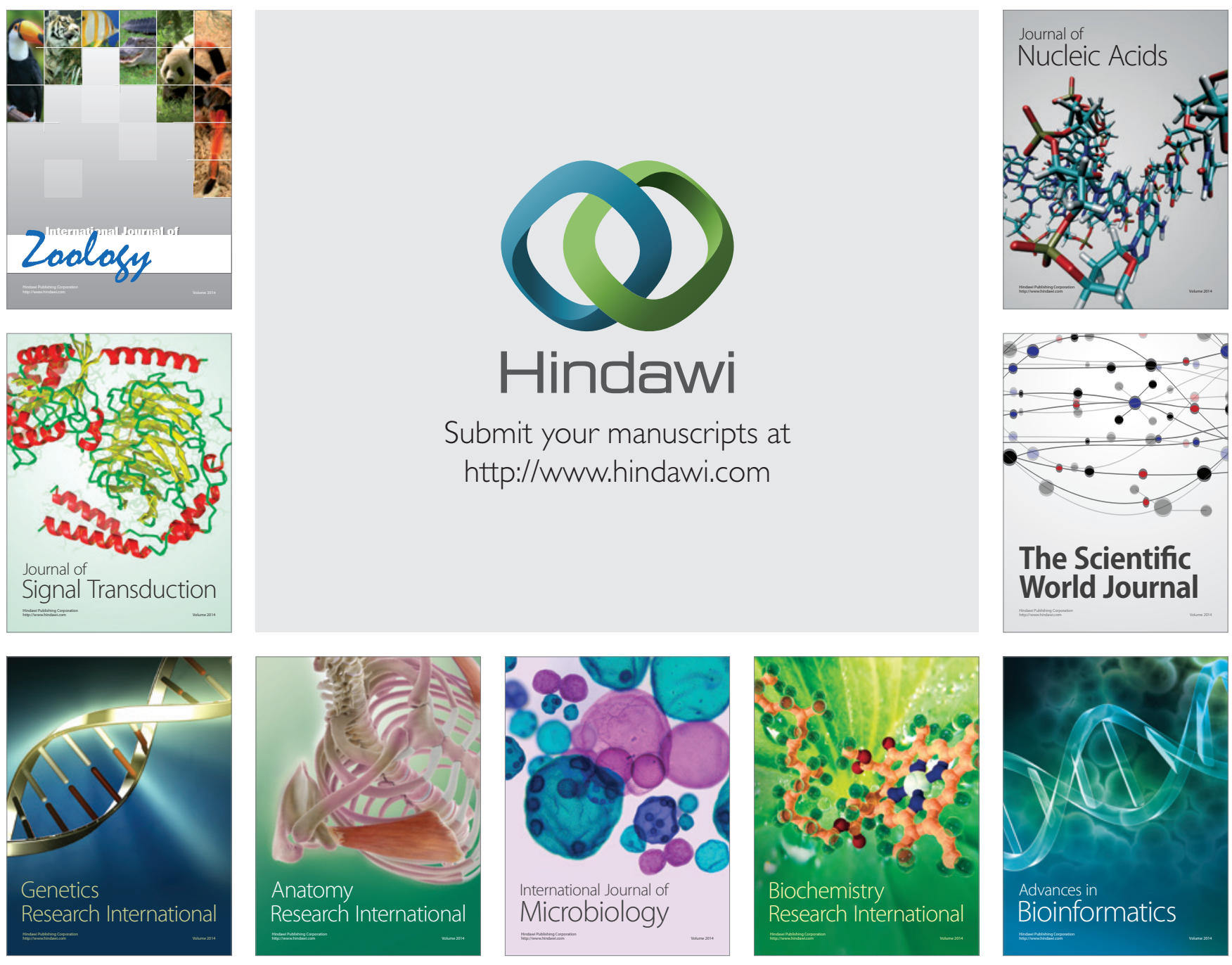

The Scientific World Journal
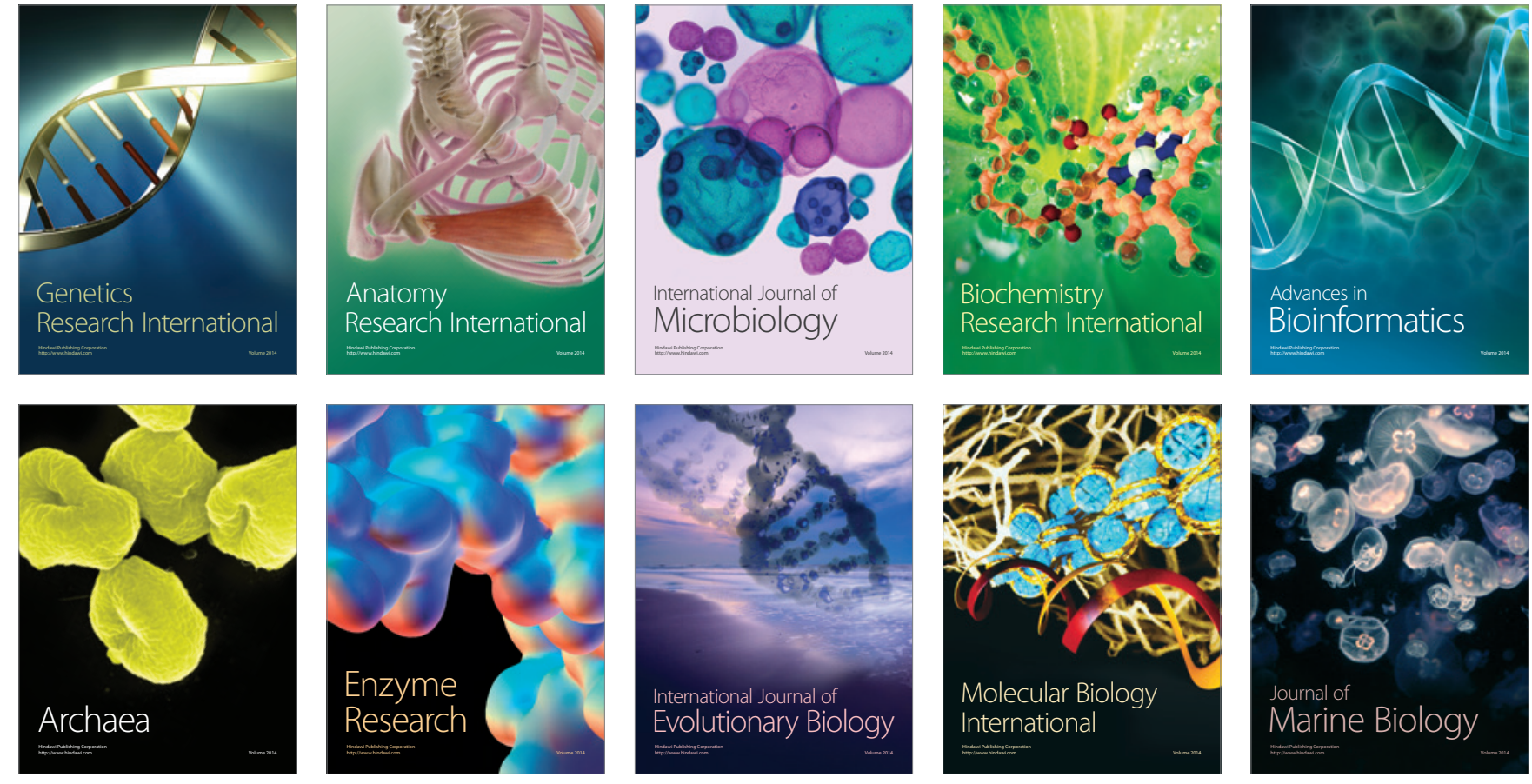